PALEO

Revue d'archéologie préhistorique

$30-2$ | 2020

Varia

\title{
Jean Combier
}

(1926-2020)

Jean-Philippe Rigaud et Jean-Paul Thévenot

\section{CpenEdition}

Journals

Édition électronique

URL : http://journals.openedition.org/paleo/5008

DOI : $10.4000 /$ paleo.5008

ISSN : 2101-0420

Éditeur

SAMRA

\section{Édition imprimée}

Date de publication : 1 septembre 2020

Pagination : 11-12

ISSN : 1145-3370

Référence électronique

Jean-Philippe Rigaud et Jean-Paul Thévenot, « Jean Combier », PALEO [En ligne], 30-2 | 2020, mis en ligne le 30 septembre 2020, consulté le 10 décembre 2020. URL : http://journals.openedition.org/ paleo/5008; DOI : https://doi.org/10.4000/paleo.5008

\section{(ब) $\odot \Theta$}

PALEO est mis à disposition selon les termes de la licence Creative Commons Attribution - Pas d'Utilisation Commerciale - Pas de Modification 4.0 International. 


\section{JEAN COMBIER $(1926-2020)$}

Jean Combier nous a quitté le 12 mars 2020, dans sa $94^{\mathrm{e}}$ année. Avec lui disparaît un préhistorien de renom appartenant à cette génération qui a marqué la seconde moitié du XXe siècle en renouvelant les bases scientifiques et méthodologiques de notre discipline que ce soit sur le terrain ou en laboratoire. Il a fait connaître largement les résultats de ses recherches et s'est attaché à étudier, à protéger et à mettre en valeur des sites qui sont maintenant devenus des références mondiales.

Né en 1926 à Lamure-sur-Azergues (Rhône), Jean Combier a fait ses études secondaires au Lycée du Parc de Lyon et malgré une adolescence marquée par la guerre, il a manifesté très tôt un vif intérêt pour la préhistoire locale, initié par la lecture du « Mâconnais préhistorique » d'H.de Ferry et A. Arcelin (1870).

Il est entré au CNRS comme collaborateur de M. Thoral directeur du laboratoire de géologie de l'Université de Lyon qui lui a confié l'étude des collections des fouilles réalisées dans le gisement de Solutré entre 1907 et 1925 par H. Breuil et A. Arcelin notamment, mais aussi par d'autres fouilleurs comme Depéret par exemple, et qui fut publiée en 1955. Nommé directeur de recherche en 1985, il a dirigé alors le laboratoire de Préhistoire méditerranéenne et rhodanienne (UPR 46) jusqu'en 1992. Il a exercé parallèlement les fonctions de Directeur des Antiquités préhistoriques de la région Rhône-Alpes et présidé en 1983 la Conférence des Directeurs des Antiquités. Il a été membre du Conseil Supérieur de la Recherche archéologique de 1964 à 1984 et membre du Comité National du CNRS et fit partie de la Commission supérieure des Monuments historiques section Grottes ornées -, de 1977 à 1995.

Jean Combier a soutenu sa thèse à l'Université de Paris VI en 1963 ; elle fut publiée dans les Mémoires de l'Université de Bordeaux en 1967. Il a été chargé d'enseignements de la Préhistoire paléolithique aux universités de Lyon 1 et Lyon 2, à l'École Pratique des Hautes études et au Muséum National d'Histoire Naturelle de Paris. Auteur de plus de 200 publications, il a été à l'origine de la création des Musées de Préhistoire d'Orgnac-l'Aven et de Solutré et fondateur de la revue Études Préhistoriques.

Le prix d'Anthropologie de l'Académie des Sciences lui a été décerné en 1966. Il était Officier dans l'Ordre des Arts et lettres (1979) et Chevalier de la Légion d'Honneur (1983).

Dès le début de sa carrière, Jean Combier s'est intéressé aux gisements du nord du bassin du Forez situés dans les gorges de la Loire, déjà connus de J. Déchelette et de nombreux préhistoriens dont J.-L. Porte qui collabora à ses recherches. Elles portèrent entre autres sur les gisements

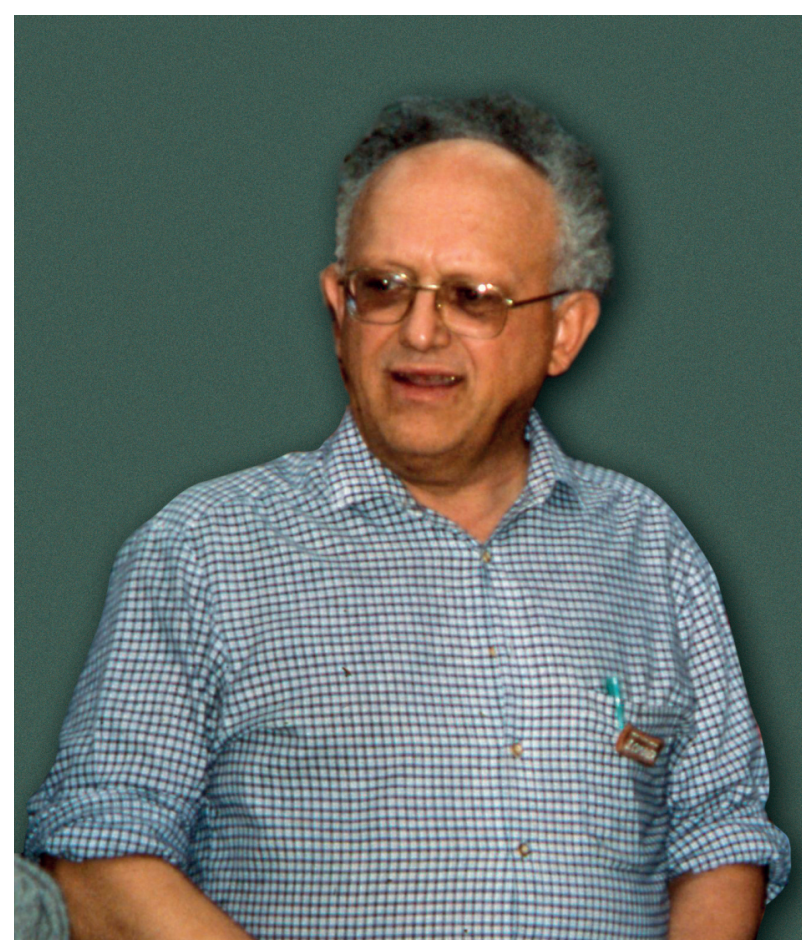

Jean Combier - colloque de Roanne-Villerest, juin 1982 (cliché J.-Ph. Rigaud).

du Champ-Grand en 1956, du Rocher de la Caille en 1966. Cette activité archéologique et les découvertes qui en résultèrent furent à l'origine de la création à Roanne du Centre départemental de recherche et de documentation préhistorique sous l'autorité de la Direction des Antiquités Préhistoriques; y furent regroupés, outre le produit des fouilles des deux sites précédents, celui de la Goutte Roffat et surtout de La Vigne Brun issus des fouilles de sauvetage qui eurent lieu de 1977 à 1983 lors de la création du barrage de Villerest. Ces importantes collections ont été, pour certaines, déposées dans les musées régionaux (Mâcon, Roanne) et pour d'autres - Champ-Grand, Grotte Roffat, La Vigne Brun, Rocher de la Caille -, données à l'État, et sont maintenant conservées au Musée national de Préhistoire des Eyzies.

La découverte, en 1866 au pied de la Roche de Solutré, d'un gisement préhistorique devenu le site éponyme du Solutréen, avait attiré les plus grands noms de la Préhistoire, mais la rusticité des méthodes de fouilles anciennes ne permettait pas de répondre aux interrogations que posait ce site magnifique. Devant ce constat, Jean Combier entreprit, à partir de 1966, des sondages et des fouilles dans un site particulièrement bouleversé. Il parvint en 1973 à mettre au jour un témoin en place du niveau solutréen qu'il fouilla sur moins de 10 mètres carrés. Ses fouilles, menées de 1968 à 1976, lui ont permis de reconnaître la structure du gisement formé de deux cônes d'éboulis imbriqués dont le plus récent niveau, daté du Magdalénien récent, a été fouillé sur une centaine de mètres carrés. Les travaux de Jean Combier ont été poursuivis par ceux de J.-L. Porte et B. Gély en 1986-1987 et par J. Hofman et A. Monthet-Wite en 1997-1998. Les objectifs de ces fouilles étaient cependant en partie atteints s'agissant de recueillir les vestiges paléontologiques et archéologiques dans un contexte stratigraphique précis et 
de faire les prélèvements nécessaires pour des datations radiométriques. Un ouvrage a été publié en 2016 sous la direction de Jean Combier ; il fait une synthèse utile sur ce site emblématique à différents égards.

Le Vivarais a été une région privilégiée dans les recherches de Jean Combier. Les nombreuses grottes des gorges de l'Ardèche ont en effet donné la matière à sa thèse soutenue en 1963. En 1964, il a entrepris des fouilles dans le gisement d'Orgnac 3, JC écrivait le plus souvent Orgnac 3), un vaste aven du plateau calcaire qui borde les gorges de l'Ardèche, sur la commune d'Orgnac-l'Aven. Dans les niveaux attribués au Riss, les industries acheuléennes ont montré dans les niveaux supérieurs l'émergence d'une production Levallois associée à de nombreux racloirs qui ont incité Jean Combier à évoquer un Prémoustérien. Dans ces niveaux acheuléens, des concentrations de produits de débitage et des foyers attestent une organisation élémentaire de l'espace.

Les grottes ornées des gorges de l'Ardèche ont été un domaine de recherche que Jean Combier a particulièrement approfondi. Dans le cadre de sa mission de directeur des Antiquités, il s'est beaucoup investi, avec l'aide de ses proches collaborateurs J.-L. Porte, P. Ayrolles et B. Gély, dans la protection, la conservation et l'étude de ces témoins très menacés. Ils ont entrepris un travail important d'inventaire systématique et de relevés photographiques des parois décorées et réalisé ainsi un état des lieux rigoureux et détaillé qui a honorablement contribué à l'atlas des grottes ornées paléolithiques françaises " l'Art des Cavernes », ouvrage collectif édité en 1994 par le Ministère de la Culture.
Les caractères stylistiques des représentations pariétales incitaient naturellement à différencier une province rhodanienne distincte de l'ensemble franco-catabrique. Tout en reconnaissant les particularités stylistiques ardéchoises, Jean Combier s'est attaché à montrer que des affinités stylistiques avec les grottes du Quercy, Cougnac et Pech-Merle, suggéraient de possibles contacts solutréens. De même, des liens entre l'Ardèche et les Pyrénées semblent bien montrer que l'Ardèche appartient au domaine franco-cantabrique sans renoncer à ses traits régionaux caractéristiques.

En tant que directeur des Antiquités, mais aussi à titre personnel, Jean Combier s'est toujours intéressé à la Préhistoire récente et à l'âge du Bronze ; il s'est efforcé d'en développer l'étude en encourageant l'exécution de fouilles scientifiques par exemple dans les coffres et dolmens ardéchois ou les gisements néolithiques de la vallée de la Saône. Mais il a lui-même publié des articles sur un gisement néolithique du Forez, des armatures de flèches du Néolithique final de la Drôme ou encore des épingles à tête décorée du Bronze final des vallées de la Saône et du Rhône. Son intérêt pour le passé l'a amené aussi à des sujets d'arts et traditions populaires comme les poteries dites du Val de Saône ou les mortiers domestiques des siècles très récents qu'il traitait avec le même esprit scientifique que des objets d'étude archéologique.

Jean-Philippe RIGAUD et Jean-Paul THÉVENOT 